\title{
Pagane Bildung im christlichen Byzanz: Basileios von Kaisareia, Michael Psellos und Theodoros Metochites
}

\author{
KARIN METZLER
}

Wie stellte sich Byzanz zum ererbten griechisch-heidnischen Bildungsgut der klassischen Antike ${ }^{1}$ Exemplarisch für dieses große Thema möchte ich - nach einer Einführung in die Problemstellung - drei Autoren aus früh-, mittel- und spätbyzantinischer Zeit vorstellen.

Als sich das Christentum durchsetzte, stand es in Spannung zur umgebenden paganen Kultur: Zwar fand es auch deshalb Anhänger, weil das Neue Testament allgemeinverständlich war, doch für Gebildete musste diese Schrift eher peinlich sein, weil sie den literarischen Maßstäben nicht entsprach, die man an ein ernst zu nehmendes Werk stellte. ${ }^{2}$ Die griechische Übersetzung des Alten Testaments wiederum mutete Gebildeten archaische Mythen wie etwa den Schöpfungsbericht zu. Wer das Christentum angreifen wollte, etwa Kaiser Julian, verfehlte denn auch nicht, solche Peinlichkeiten herauszustreichen. ${ }^{3}$

Für den christlichen Intellektuellen gab es zwei Wege der Verteidigung: Er konnte entweder sagen: „Ja, unser Testament ist kein Werk der hohen Literatur, und das ist gerade das Gute daran“, oder aber: „Wer das sagt, hat die Bibel nicht verstanden. Jeder Satz, jedes Wort ist voll des tiefsten Sinns, man muss ihn nur zutage fördern." Er konnte also entweder den Vorwurf akzeptieren, aber zur Auszeichnung erklären - oder den Gegenbeweis antreten. In der erhaltenen patristi-

1 Es handelt sich um die überarbeitete Fassung des Vortrags, den die Verfasserin am 14. 1. 2004 zu ihrer Habilitation im Fach Byzantinistik an der Freien Universität Berlin gehalten hat.

$2 \mathrm{Zu}$ den Schwierigkeiten gebildeter Christen mit dem biblischen Stil E. Norden, Die antike Kunstprosa vom VI. Jahrhundert v. Chr. bis in die Zeit der Renaissance, Darmstadt ${ }^{5} 1958$, II 516-534 und I. Ševčenko, Levels of Style in Byzantine Prose, in: JÖB 31/1 (1981) 289-312, hier 298f. Ein sprechendes Beispiel ist Isid. Pel. Ep. 1555 (IV 28; PG 78, 1080D-1081A) = P. Évieux, Isidore de Pélusion. Lettres 1414-1700 (SC 454), Paris 2000, II 244-246.

3 Z. B. Iulian. imp. Contra Galilaeos Fr. 9.13-17 Masaracchia. Nach Or. Cels. III 44, 104,4-5 Borret unterstellte man den Christen als Motto „Kein Gebildeter trete hinzu“. Mit Vorwürfen gegen die Heilige Schrift setzt sich auseinander: Isid. Pel. Ep. 1412 (IV 67; PG 78 1124B 1125 A) = P. Évieux, Isidore de Pélusion. Lettres 1214-1413 (SC 422), Paris 1997, I 498-500 Évieux; 1697 (IV 91; PG 78 1152A - 1153B) II 458-62 Évieux. 
schen Literatur überwiegt die Strategie, die Weisheit des biblischen Wortes zu beweisen; dazu entfaltete man die Methoden der allegorischen Auslegung; die Scholien der Homer-Philologie entwickelten sich zum durchlaufenden Bibelkommentar, in dem jedes Wort, jeder Satz auf seinen tieferen Sinn hin abgetastet wurde. ${ }^{4}$ Man behauptete (und übernahm damit ein jüdisches Argument): Wo die pagane Literatur von ähnlicher Tiefe ist, ist sie abhängig von der Bibel; z. B. hat Platon seine Lehrsätze von Mose abgeschrieben, ${ }^{5}$ das griechische Epithalamion ist abhängig vom Hohen Lied Salomos. ${ }^{6}$ Selbst stilistisch wurde sie verteidigt. ${ }^{7}$ Der klassische Maßstab war damit anerkannt. Eine solche Haltung passte in ein Christentum, in der die innerkirchliche Auseinandersetzung zu einem Ringen um die Ausbildung einer philosophisch stringenten Lehre führte; dies begann im 4. Jahrhundert mit dem Arianischen Streit. ${ }^{8}$ Dies enthob auch eines Problems beim Verfassen der eigenen theologischen Literatur: Selbst im „Fischerstil“ des Neuen Testaments zu schreiben, fiel nämlich denen schwer, die durch die Schule der spätantiken Bildung gegangen waren. ${ }^{9}$

Man konnte dagegen, wie gesagt, den Makel der literarisch minderwertigen Bibel auch in einen Vorzug ummünzen; Beispiele dafür sind vor allem aus der westlichen Theologie erhalten; Augustin etwa rühmte den niedrigen Stil, den „sermo humilis“, der die Wahrheit zu den einfachsten Menschen bringe; die hochgestochene nicht-christliche Literatur desavouierte sich in seinen Augen

4 Vgl. Ševčenko, Levels (s. Anm. 2), 229: Photius beurteilt Paulus nach paganem Stil-Maßstab.

5 R. Klein in: Gregor der Wundertäter, Oratio prosphonetica ac panegyrica in Origenem / Dankrede an Origenes. Im Anhang: Origenis epistula ad Gregorium Thaumaturgum / Der Brief des Origenes an Gregor den Wundertäter. Übersetzt von P. Guyot. Eingeleitet von R. Klein (Fontes Christiani 24), Freiburg/Basel/Wien/Barcelona/Rom/New York 1996, 101: Die Griechen lernten von Mose, bzw. Gott schenkte den Griechen besondere Erkenntnis (vgl. W. Jaeger, Das frühe Christentum und die griechische Bildung, Berlin 1963, 45).

6 Origène, Commentaire sur le Cantique des Cantiques.Tome I. Texte de laversion Latine de Rufin. Introduction, traduction et notes par L. Brésard/H. Crouzel (SC 395), Paris 1991, prol. 1,1: Epithalamium libellus hic, id est nuptiale carmen.

7 Norden, Kunstprosa (s. Anm. 2), 526-528.

8 Der Arianische Streit hätte sich auch auf sermo-humilis-Ebene austragen lassen (biblizistisch z. B. Cyrillus Hierosolymitanus, Eusebius Emesenus), aber die Theologie argumentierte weitgehend auf philosophischer Ebene. - In den Auseinandersetzungen um Konzilsentscheidungen spielte auch später noch das Argument eine Rolle, bestimmte Termini gehörten nicht in die Sprache der Fischer. Zur Bedeutung der ,humanistisch“ geprägten Theologie in Byzanz: G. Podskalsky, Von Photios bis Bessarion. Der Vorrang humanistisch geprägter Theologie in Byzanz und deren bleibende Bedeutung (Schriften zur Geistesgeschichte des östlichen Europa 25), Wiesbaden 2003.

9 Norden, Kunstprosa (s. Anm. 2), 529. So gebildet waren freilich nicht alle, die sich zum Christentum bekannten; wie weit unter diesen Lesen und Schreiben verbreitet war, ist ein Problem: R. L. Fox, Literacy and Power in Early Christianity, in: A. K. Bowman/G. Woolf (Hrsgg.), Literacy and Power in the Ancient World, Cambridge 1994, 126-148, hier 144: Fox unterscheidet Nicht-lesen- und Nicht-schreiben-Können und führt als Beleg Lektoren, die nicht schreiben können, und (135) die Möglichkeit eines illiteraten Bischofs nach der syrischen Didascalia an. 
durch ihren Elitarismus. ${ }^{10}$ Auch im Osten gab es diese Haltung: Sie lässt sich etwa am Bildungsgang der Helden vieler Heiligenviten ablesen. ${ }^{11}$ Die Ablehnung von paganer Bildung und Literatur in bestimmten Kreisen setzte sich in späterer Zeit als Indifferenz fort, etwa im Hesychasmus. Für die ausdrückliche Polemik der spätantiken Zeit ${ }^{12}$ hier nur eine Kostprobe aus einer frühen Kirchenordnung, der syrischen Didaskalia, in der Übersetzung von Georg Schöllgen: ${ }^{13}$

„Von allen Schriften der Heiden (...) halte dich fern. (...) Was fehlt dir denn am Wort Gottes, daß Du dich auf diese Fabeln der Heiden stürzt? Wenn Du Geschichtsberichte lesen willst, so hast Du das Buch der Könige, wenn aber Weise (...), so hast Du die Propheten, bei denen Du mehr Weisheit und Einsicht findest, als (bei den) Weisen und Philosophen (...); denn es sind die Worte des einen, allein weisen Gottes. Und wenn Du Lieder begehrst, so hast Du die Psalmen Davids, und wenn Gesetze und Gebote, so hast Du das Gesetz (...) des Herrn. Aller fremden (Schriften) also (...) enthalte dich gänzlich. ${ }^{\text {(14 }}$

Wenn man in dieser Weise argumentierte, war die Verurteilung der paganen Literatur klar; wenn man aber die Wertmaßstäbe der klassischen Literatur anerkannte, um für die Bibel Anspruch auf sie zu erheben, wurde zum Problem, wie man sich zu dieser Literatur selbst stellte. Genau zu dieser Frage führt der erste Text, der hier vorgestellt werden soll.

10 Der paradoxe Befund, dass sich in der Ostkirche nie Klosterkirchen für die Erziehung von „,weltlichen“ Schülern etablierten (H.-I. Marrou, Geschichte der Erziehung im klassischen Altertum, hrsg. von R. Harder, Freiburg im Breisgau 1957, 477-481), im Westen aber die Klosterschule zentraler Bildungsort wurde (ebd. 481-490), hängt vielleicht genau am Misstrauen gegen die pagane Bildung und damit auch ihr Bildungssystem.

11 S. Rubenson, Philosophy and Simplicity. The Problem of Classical Education in Early Christian Biography, in: T. Hägg/P. Rousseau/C. Høgel (Hrsgg.), Greek Biography and Panegyric in Late Antiquity, Berkeley/Los Angeles/London 2000, 110-139 (bes. 115-119); dies beginnt gleich mit dem ersten Vertreter der Gattung: Athanase d'Alexandrie, Vie d'Antoine. Introduction, texte critique, traduction, notes et index par G. J. M. Bartelink (SC 400), Paris 1994, 1,1f. (130f.): Antonios wehrt sich bereits gegen die Elementarbildung. Es konnte aber auch in der Heiligenvita die Bildung einbezogen werden.

12 So auch Tatian und Tertullian nach R. Klein, Die Bedeutung von Basilius' Schrift ,ad adolescentes" für die Erhaltung der heidnisch-griechischen Literatur, in: Roman Quarterly 92 (1997) 162-176, hier 163f. (zu Tertullian auch G. Schöllgen, Die Anfänge der Professionalisierung des Klerus und das kirchliche Amt in der syrischen Didaskalie [JbAC Ergänzungsband 26], Münster 1998, 177 Anm. 26). Beispielgestalten sind Schenute und Epiphanios.

13 Schöllgen, Anfänge (s. Anm. 12), 176f. Schöllgen weist darauf hin, dass es für die durch die pagane Bildung geprägte Oberschicht ein kleines Opfer war, sich der paganen Literatur zu enthalten, was er vor allem mit der syrischen Didaskalie belegt (zu dieser auch Klein, Bedeutung [s. Anm. 12], 164), aber auch mit Verweis auf Hieronymus,ep.22, 30 (176 Anm. 22). Vgl. auch Marrou, Geschichte (s. Anm. 10), 464.

14 Schöllgen, Anfänge (s. Anm. 12), 176f. 
Um das Jahr $370^{15}$ legt der Bischof von Kaisareia, Basileios, seine Stellungnahme zur paganen Literatur in einer Grundsatzschrift nieder. In einem Brief wendet er sich an seine Neffen ${ }^{16}$ und legt dar, wie junge Christen mit der paganen Literatur umgehen sollen. Es ist eine Schrift, wie sie Schulmeister erfreut: Zwar ist einzig das künftige Leben von Wert, und darauf führen die christlichen heiligen Schriften hin, doch ist ein junger Mensch noch nicht fähig, die Tiefe ihrer Weisheit zu verstehen; er beschäftigt sich daher am besten mit verwandter Literatur, ${ }^{17}$ das heißt: Literatur mit einem ähnlichen ethischen Anspruch. Man findet solche Literatur durchaus bei den paganen Autoren, wenn man nur die richtige Auswahl $1^{18}$ trifft. Was Basileios da auswählt, führt er an Beispielen vor: ${ }^{19}$

Homer etwa ist ein einziges Lob der Tugend, ${ }^{20}$ Solon und Theognis warnen davor, den Reichtum zu wichtig zu nehmen. ${ }^{21}$ Für die Erziehung lassen sich auch antike Tugend-Anekdoten nutzen, etwa die von Perikles, dem den ganzen Tag ein Kritiker gefolgt ist, der ihn mit Schmähungen belästigte; Perikles hat seine Philosophie schon dadurch erwiesen, dass er die Schmähungen nicht beachtete; am Schluss des Tages aber geleitet er seinen Gegner nach Hause, damit dieses unschätzbare Trainingsfeld ( $\gamma v \mu v \alpha ́$ orov) für die Hinleitung zur Philosophie keinen Schaden nehme. ${ }^{22}$

Es wird also ausschließlich nach der Ethik des Inhalts ausgewählt, nicht nach der Vollendung der Form. Die literarische Form wird gänzlich übersehen: Es sind

15 Saint Basil on the Value of Greek Literature edited by N. G. Wilson, London 1975 (= Basil. Caes. De legendis gent. libr. ed. Wilson), 9: Mitte oder Ende der 370er Jahre. (Wilson verwendet in seiner Ausgabe den Text von F. Boulenger, Aux jeunes gens sur la manière de tirer profit des lettres helléniques, Paris 1935; die neuere Ausgabe von Naldini ist mir während der Druckvorbereitung nicht zugänglich.) Anders A. Moffatt, The Occasion of St Basil's Address to Young Men, in: Antichthon 6 (1972) 74-86, hier 83: zwischen 17.6.362 (Rhetorenedikt) und 26.6.363 (Tod Julians). Nach Moffatt wäre Basileios' Schrift eine unmittelbare Antwort auf das Rhetorenedikt gewesen; doch während der Gültigkeit des Edikts war es nicht sinnvoll, die jungen Leute auf etwas Verbotenes auszurichten. Die Schrift ist nur sinnvoll vor dem Hintergrund der Wahlfreiheit der Christen, also nach dem Tode Julians. - Der Ausschluss des Zeitraums 364-370 hängt davon ab, von wann an, $d$. h in welchem Alter und ob erst mit dem Bischofsamt, man Basileios die nötige Autorität zuspricht.

$16 \mathrm{Ob}$ es seine Neffen oder andere junge Verwandte sind, scheint manchen Forschern fraglich; die Formulierung Basil. Caes. De legendis gent. libr. 8-10 Wilson (s. Anm. 15) $\tau \tilde{\eta} \tau \varepsilon \tau \alpha \varrho \dot{\alpha} \tau \tilde{\eta} \varsigma$

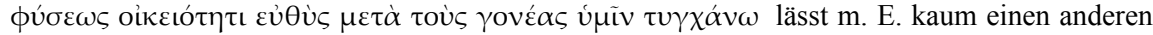
Schluss als Neffen zu; vorsichtig etwa Klein, Bedeutung (s. Anm. 12), 168 Anm. 19 nach Lamberz und Naldini.

17 Basil. Caes. De legendis gent. libr. cap. II Wilson (s. Anm. 15).

18 Basil. Caes. De legendis gent. libr. cap. I 27; VIII 2-3 Wilson (s. Anm. 15).

19 Basil. Caes. De legendis gent. libr. cap. IV-V Wilson (s. Anm. 15).

20 Basil. Caes. De legendis gent. libr. V 26-27 Wilson (s. Anm. 15). Parallelen für Lob des Odysseus führt an: V. Pyykkö, Die griechischen Mythen bei den großen Kappadokiern und bei Johannes Chrysostomos (Annales Universitatis Turkuensis 193), Turku 1991, 36.

21 Basil. Caes. De legendis gent. libr. V 47-54 Wilson (s. Anm. 15).

22 Basil. Caes. De legendis gent. libr. IV 5-11 Wilson (s. Anm. 15). 
die geschilderten Personen, denen Basileios wie Gesprächspartnern begegnen will, nicht Autoren, die zu bestimmten Zwecken Figuren gestalten. ${ }^{23}$ Was von der paganen Literatur akzeptiert wird, wird um des Nutzens willen akzeptiert: als erzieherischer Ansporn zur Tugend. ${ }^{24}$ Die unmoralischen Göttergeschichten sind ein Beispiel für das, was aussortiert werden muss ${ }^{25}$ (Basileios ist direkt abhängig von den Argumenten, die Platon dafür anführt ${ }^{26}$ ), für die Bühne hat er nur Verachtung, ${ }^{27}$ Rhetoren sind vor allem Lügner. ${ }^{28}$

Sein Standpunkt ist aller Ehren wert, doch äußerst nüchtern. Man stelle sich vor, zur Rechtfertigung des Unterrichts von Alten Sprachen auf dem Gymnasium würde angeführt, das wirke als Anfeuerung zur Tugend! $!^{29}$ Auch wir hätten zwar nichts einzuwenden, wenn Schüler sich mit Selbstkritik anfreundeten oder den Prestigewert von Markenkleidung und Mobiltelefone relativierten, aber dazu stehen die antiken Werke nicht auf dem Lehrplan. Wenn wir mit ihnen Homer lesen, dann als Kunstwerk oder Zeugnis einer vergangenen Epoche, nicht als Vehikel von Moral. Wenn wir mit ihnen Platon lesen, kommt es uns nicht auf den Sokrates an, der sich christlich-widerspruchslos schlagen lässt, ${ }^{30}$ sondern den, der so lange weiterfragt, bis er den Gesprächspartner in die Aporie geführt hat.

Als Zeugnis der Auseinandersetzung mit der klassischen griechischen Literatur bleibt die Schrift Пoò toù véouৎ des Basileios hinter ihrem Gegenstand zurück. Um ihr gerecht zu werden, muss man sich klar machen, auf welche Situation die Schrift antwortet. Basileios spricht in keiner Stunde Null, in der das Christentum ohne Voraussetzungen seine Stellung zum paganen Erbe festlegen könnte. Es gab nicht nur die geschilderten Bedenken von christlicher Seite, es hatte von Seiten des Heidentums das Verbot gegeben, dass christliche Lehrer christliche Schüler mit Homer und Platon erzogen, nämlich in dem so genannten

23 Nach Basil. Caes. De legendis gent. libr. II 37-38 Wilson (s. Anm. 15) will Basileios sich mit Dichtern, Schriftstellern, Rednern und allen Menschen unterhalten.

24 Es handelt sich also um reine Protreptik, was sich auch in der Wortwahl spiegelt: Basil. Caes.

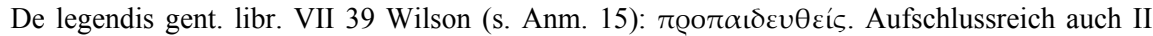

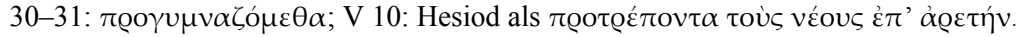

25 Basil. Caes. De legendis gent. libr. IV 19-28 Wilson (s. Anm. 15).

26 Basil. Caes. De legendis gent. libr. IV 19-28 Wilson (s. Anm. 15), abhängig von Plato R. publ. 377E-78E, 390AB, 395D, 396CD (nach Wilson, s. genauer Pyykkö, Mythen [s. Anm. 20], 34; zu den Vorbildern für Basileios' Götterkritik s. auch Klein, Bedeutung [s. Anm. 12], 171 mit Anm. 25).

27 Basil. Caes. De legendis gent. libr. IV 28 Wilson (s. Anm. 15).

28 Basil. Caes. De legendis gent. libr. IV 30-31 Wilson (s. Anm. 15).

29 Die Berechtigung der antiken Literatur wird mit ihren oberflächlichen Parallelen zu biblischen Geboten begründet: Basil. Caes. De legendis gent. libr. cap. IV Wilson (s. Anm. 15); z. B. 40ff. Alexander und die Tochter des Dareios.

30 Basil. Caes. De legendis gent. libr. VII 23-30 Wilson (s. Anm. 15); Parallele zum neutestamentlichen Gebot, die andere Wange hinzuhalten, V 31-35. 
Rhetorenedikt des Kaisers Julian von $362 .{ }^{31}$ Davon waren zwar erst einmal nur die Christen betroffen, die im Unterrichtssystem als Lehrer etabliert waren, christliche Eltern waren nicht gehindert, ihre Kinder im paganen Bildungssystem auf den Staatsdienst vorzubereiten. ${ }^{32}$ Aber die Kirche sah es als Signal und ersten Schritt zum gänzlichen Ausschluss der Christen aus der Funktionselite des Staates ${ }^{33}$ und reagierte entsprechend heftig. ${ }^{34}$ Darauf wären die Pläne Julians auch hinausgelaufen, wenn er nicht durch seinen Tod gehindert worden wäre, vor allem das geplante Gesetz zur verbindlichen Teilnahme der Beamten am Opferkult.

Mit Julians Tod war die Gefahr für die Christen, von Staats wegen von der Integration in die spätantike Kultur und Gesellschaft ausgeschlossen zu werden, gebannt. Jetzt kam es darauf an, dass sich nicht die christlichen Rigoristen durchsetzten, die die Integration von christlicher Seite ausschließen wollten. ${ }^{35}$ Ihnen begegnete man am besten mit unanfechtbarer Moral. Sie verwies man am besten auf die Übereinstimmung zwischen den antiken Tugend-Exempeln und neutestamentlichen Geboten. Basileios beugte einer Selbstzensur der Christen in der wieder gewonnenen Freiheit vor, am klassischen Bildungsweg teilzunehmen; stattdessen schlug er eine Scheidung zwischen dem Kanon des nutzbaren paganen Erbes und dem Erbe vor, das ein Christ lieber ausschlug. Damit befand er sich auf gesichertem Boden: Die unmoralischen Göttergeschichten bei Homer etwa waren ein Erbe, das auch der gebildete Heide der Spätantike lieber ausschlug und z. B. durch allegorische Deutung entschärfte. Es ist also kein Zufall, dass Basileios die Frage gerade als Erziehungsproblem aufrollte. ${ }^{36}$

31 Iulian. imp. ap. Cod. Theodos. XIII 3,5 (v. 10-15 = Cod. Iustin. X 53,7) (17. Juni 362). Ausführungsbestimmungen: Lettres et fragments. Texte revu et traduit par J. Bidez, Paris 1960, ep. 42 (73,1-15). Julian argumentierte geschickt, weshalb die Christen selbst die Einbindung in die pagane Bildung ablehnen müssten. Zur Datierung bei Moffatt, Occasion (s. Anm. 15) in unmittelbarem Zusammenhang mit dem Rhetorenedikt.

32 C. Hardy, Kaiser Julian und sein Schulgesetz, in: R. Klein (Hrsg.), Julian Apostata (WdF 509), Darmstadt 1978, 387-408, wendet sich gegen die communis opinio, es sei das Ziel gewesen, öffentliche Schulen von christlichen Mitgliedern zu säubern, und bezweifelt (388), dass der Schulbesuch christlicher Kinder verboten wurde; er kennt aber auch (401 Anm. 7) zwei Belege dafür, dass christliche Lehrer ihre Lehrstühle aufgaben. - Für die Bedeutung der Bildung für das Vorankommen im Beamtentum im 5. Jh. s. Cod. Theodos. XIV 1,1 Mommsen.

33 A. P. Agapitos, Teachers, Pupils and Imperial Power in Eleventh-century Byzantium, in: Y. L. Too/N. Livingstone (Hrsgg.), Pedagogy and Power. Rhetorics of Classical (Ideas in context 50), Cambridge 1998, 170-191, hier 172, spricht von „social extinction“.

34 Z. B. Greg. Naz. Or. 4 contra Iulianum ed. Bernardi.

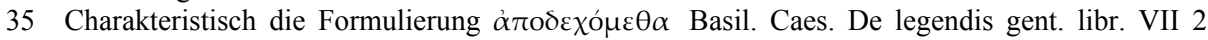
Wilson (s. Anm. 15). - Zum Erfolg der Schrift des Basileios s. L. Schucan, Das Nachleben von Basilius Magnus „Ad adolescentes“. Ein Beitrag zur Geschichte des christlichen Humanismus, Genf 1973, 42-53; Klein, Bedeutung (s. Anm. 12) arbeitet die Bedeutung der Schrift für die Erhaltung der paganen griechischen Literatur heraus.

36 Die Zweckbestimmung wird hier ähnlich wie von Boulenger (referiert Moffatt, Occasion [s. Anm. 15], 80) gesehen: Anlass und erste Adressaten waren die Neffen, aber ein größerer Rezi- 
Gab es also nach Basileios keine Zensur der paganen Literatur durch das herrschende Christentum mehr? Doch, es konnte zu Konflikten kommen; Basileios hatte ja nicht jeden beliebigen Gebrauch gerechtfertigt. Ein empfindlicher Punkt war die Philosophie Platons: Sie wurde einerseits in wesentlichen Teilen in das Christentum integriert, ${ }^{37}$ andererseits hatte jeder Byzantiner den Eindruck zu vermeiden, er hänge der Ideenlehre an, betreibe seine Platon-Studien als Selbstzweck. Wie alarmierend die Andeutung eines solchen Vorwurfs wirken konnte, davon haben wir ein dramatisches Zeugnis in Michael Psellos' Brief an den späteren Patriarchen Xiphilinos. Er antwortet auf einen (uns nicht erhaltenen) Brief des Xiphilinos, in dem dieser die Formulierung „dein Platon“ benutzt hatte. Psellos setzt gleich am Anfang des Briefes mit der Replik auf diese Formulierung ein:

„Platon mein?, Du Heiligster und Weisester, mein? ${ }^{38}$, o Erde und Sonne (damit auch ich meine Tragödie auf der Bühne des Wortes aufführe) $?^{39}$ Denn wenn Du damit den Vorwurf machst,$^{40}$ dass ich mich zu Zeiten oft mit dem Mann unterhielt in seinen Dialogen, das Gepräge des Satzbaus bewunderte und seine Gewalt in den Beweisen als göttlich verehrte, warum wirfst $\mathrm{Du}$ das nicht auch den Großen unter unseren $<$ Kirchen>vätern vor, die mit ihren Beweisen Leuten wie Eunomios und Apolinarios ihre Häresien widerlegten, weil sie sie mit der Schärfe ihrer logischen Schlüsse angreifen konnten? Wenn $<$ Du es $>$ aber $<$ mit dem Vorwurf verbindest $>$, dass ich seinen Lehren

pientenkreis war von Anfang an vorgesehen. Die Gegengründe bei Moffatt, Occasion 80 sind nicht stichhaltig: Die Veröffentlichung von Privatbriefen ist in der spätantiken und byzantinischen Briefkultur gerade etwas Normales.

37 H. Hunger, Die hochsprachliche profane Literatur der Byzantiner (HdAW XII, Byzantinisches Handbuch 5.1-2), München 1978, I 15-25. Vgl. H.-V. Beyer, Studien zum Begriff des Humanismus und zur Frage nach dessen Anwendbarkeit auf Byzanz und andere vergleichbare Kultu-

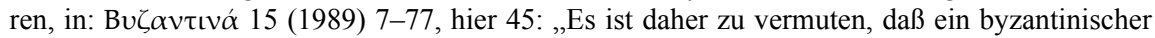
Philosoph umso mehr Gefahr lief, von der Kirche als Häretiker betrachtet zu werden, je weiter er sich vom Neuplatonismus entfernte (...).“

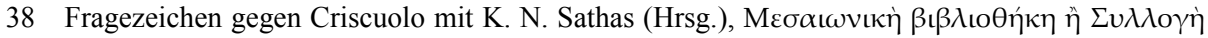

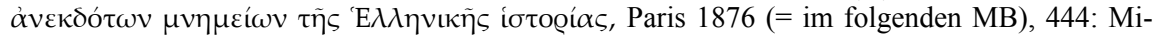
chael Psellos ep. 175 (ebenso in einer noch unpublizierten Edition von D. R. Reinsch) gegen Michele Psello, Epistola a Giovanni Xifilino. Testo critico, introduzione, traduzione e commentario a cura di U. Criscuolo (Byzantina et Neo-Hellenica Neapolitana 1), Neapel 1973, I.3 (= Michael Psellos ep. ad Xiph. Criscuolo); die Formulierung wird übrigens mehrfach fälschlich in affirmativem Sinn zitiert (Hunger, Literatur [s. Anm. 37], I 20; G. Misch, Geschichte der Autobiographie. 3. Bd.: Das Mittelalter. 2,2: Das Hochmittelalter im Anfang, Frankfurt am Main 1962, 787 mit Anm. 107; R. Browning, Enlightenment and Repression in Byzantium in the Eleventh and Twelfth Centuries, in: P\&P 69 [1975] 3-23, hier 11).

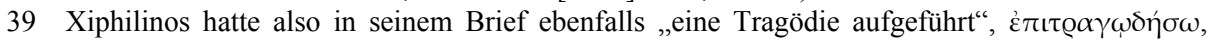
vielleicht mit einem ähnlichen Ausruf.

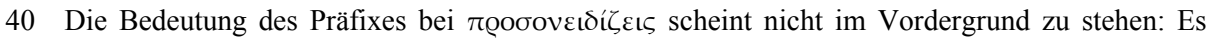
werden zwei mögliche Vorwürfe herausgelesen, s. $\mu \dot{\varepsilon} v-\delta$ ’ ŏ $\tau$. 
folge oder mich auf seine Gesetze stütze, dann hast Du keine richtige Meinung von uns, Bruder."“1

Der Schreiber reagiert im Großteil seines Briefes darauf, dass Xiphilinos von 'Deinem Platon' geschrieben hatte. Emphatisch verteidigt sich Psellos: Kein Zweifel, er bewundere Platon (und andere pagane Autoren) sehr, aber unendlich mehr bedeuteten ihm die Heilige Schrift und das Kreuz Christi; ${ }^{42}$ darum habe er sich schließlich auch zum Mönch weihen lassen. Und er kann einige Argumente anführen, das erste haben wir schon gehört: Die großen Kirchenväter Basileios, Gregor von Nyssa und Gregor von Nazianz konnten Häretiker nur deshalb widerlegen, weil sie die antiken Regeln des logischen Schlusses beherrschten. ${ }^{43}$ Hingewiesen hat Psellos auch schon im ersten Absatz auf die literarischen Vorzüge der platonischen Dialoge. Ferner führt er an: Pagane Philosophie ist eine notwendige Propädeutik, um zum Nous und darüber hinaus zu steigen. ${ }^{44}$ Außerdem hat Psellos platonische Lehren widerlegt - soweit sie sich nicht ohnehin bestens in das christliche Lehrgebäude einfügen wie die über Gerechtigkeit und Unsterblichkeit (also „Politeia“ und „Phaidon“); ${ }^{45}$ auch für anderes paganes Bildungsgut, das ihm Xiphilinos vorgehalten hat, rechtfertigt sich Psellos. Am Rande werden kleine Spitzen zwischen dem geschliffenen Konstantinopolitaner und dem (angeblichen) Provinzler aus Trapezunt ausgetauscht. ${ }^{46}$

Doch immer wieder kommt er darauf zurück, dass Xiphilinos von „Deinem Platon“ gesprochen hat. Was trifft Psellos am Platonismus-Vorwurf so tief? Er versteht ihn als Drohung mit dem, was zu anderer Zeit und an anderem Ort Inqui-

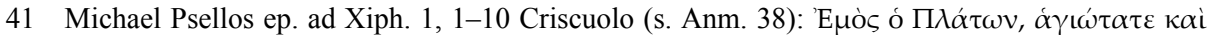

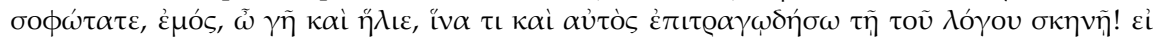

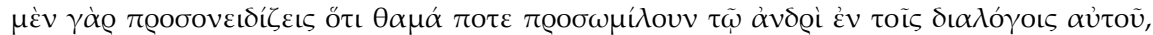

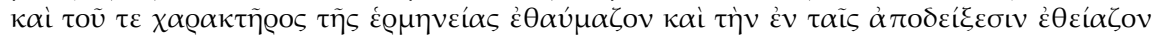

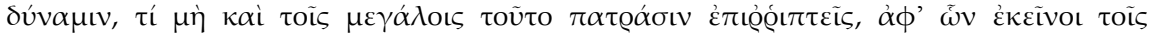

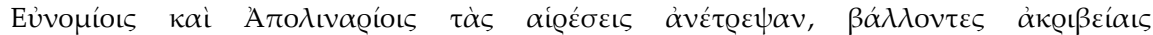

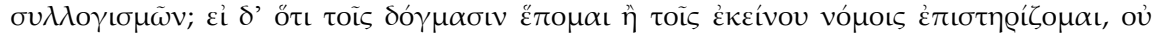

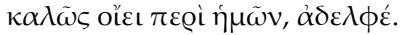

42 D. G. Dakouras, Michael Psellos' Kritik an den alten Griechen und dem griechischen Kult, in:

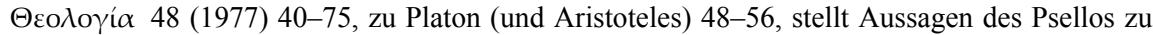
paganen Autoren mit dem Ergebnis zusammen, dass seine Kritik an ihnen die Wertschätzung überwogen habe. Psellos' affirmatives Verhältnis zu Platon beschreibt derselbe Autor: D. G. Dakouras, Die Rehabilitation der griechischen Studien im XI. Jahrhundert und Michael Psellos,

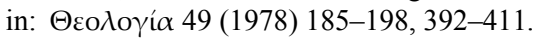

43 Agapitos, Teachers (s. Anm. 33), 182 bringt dies in Verbindung mit Psellos' Gregor-Studien.

44 Michael Psellos ep. ad Xiph. 41; 170-172 Criscuolo (s. Anm. 38).

45 Michael Psellos ep. ad Xiph. 23-26 Criscuolo (s. Anm. 38).

46 Michael Psellos ep. ad Xiph. 138-50 Criscuolo (s. Anm. 38). 
sition heißen wird: ,,.. wenn 〈Du mir〉 nicht 〈gehorchen willst〉, wirst Du Bekanntschaft machen mit Foltergeräten, von denen Du noch nie gehört hast. “47

Dass Psellos zu diesem Zeitpunkt Anlass zu einem solchen Verdacht hat, kann man bezweifeln: Wie wir noch sehen werden, hat Xiphilinos im Augenblick keine Machtposition in der Kirche, er ist noch nicht Patriarch. Doch zeigt das Exempel des Ioannes Italos, der 1082 wegen seines (angeblichen) Platonismus verurteilt werden wird, ${ }^{48}$ dass es in Byzanz durchaus gefährlich werden konnte, wenn man Platon als Eigenwert betrachtete, statt einzelne seiner Lehren in die christliche Theologie einzuordnen. Wurde man für seine philosophischen Ansichten verurteilt, wurde das allerdings mit dem Exil, nicht mit dem Tode bestraft. ${ }^{49}$

Doch der Platonismus-Vorwurf ist nicht der einzige Streitpunkt, der im Brief sichtbar wird. Schaut man sich den biographischen Hintergrund näher an, verliert das emphatische Motiv seinen Charakter als Haupt-Auseinandersetzungspunkt und gerät in die Nähe eines vorgeschobenen Arguments. Man muss sich an die Vorgeschichte der beiden Kontrahenten erinnern: Sie haben als Freunde in ihrem Studium bei Ioannes Mauropus begonnen. Seit dem Abschluss ihrer Bildung haben beide einen steilen Karriereweg genommen, der jedem von beiden um $1045^{50}$ eine kaiserliche Stiftungsprofessur ${ }^{51}$ des Kaisers Konstantin IX. Monomachos eintrug: Xiphilinos beaufsichtigte als Nomophylax die gesamte juristische Aus-

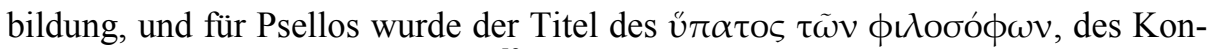
suls der Philosophen, geschaffen. ${ }^{52}$ Hier ist übrigens die Stelle, anzumerken, dass Xiphilinos nicht der Banause ist, als den ihn der Brief des Psellos hinstellt; seine

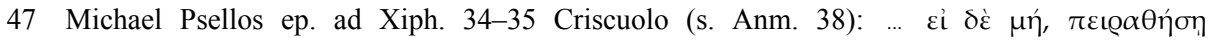

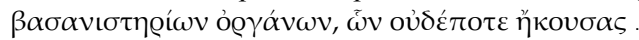

48 Browning, Enlightenment (s. Anm. 38), 11-15; entscheidend war der zweite Prozess 1082, s. ebenda 13. Der Vorwurf des Neoplatonismus sei bei Ioannes Italos nicht berechtigt: Beyer, Studien (s. Anm. 37), 45.

49 Browning, Enlightenment (s. Anm. 38) (für die Komnenenzeit; Fälle s. 18f.; Gegensatz zu populären Häresien wie dem Bogomilismus 19): „Few were exiled, no one condemned to death for his philosophical views.“ Eustratios von Nikaia z.B. kam persönlich nicht zu Schaden: Browning, Enlightenment 18 (dort auch Fälle, in denen Verurteilung zur Verbannung führte).

50 So datieren F. Fuchs, Die höheren Schulen von Konstantinopel im Mittelalter (Byzantinisches Archiv 8), Leipzig/Berlin 1926, 25; Browning, Enlightenment (s. Anm. 38), 8. Hunger, Literatur

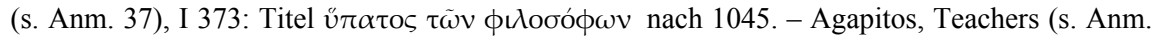
33), 178 datiert die Einrichtung der Rechtsschule auf 1047.

51 Zur Frage der angeblichen Universitätsgründung durch Konstantin Monomachos (vertreten etwa durch Fuchs, Schulen [s. Anm. 50], 24-35) in Konstantinopel: G. Weiß, Oströmische Beamte im Spiegel der Schriften des Michael Psellos (MBM 16), München 1973, 65-76.

$52 \mathrm{Zu}$ den Schulen beider s. W. Wolska-Conus, Les écoles de Psellos et de Xiphilin sous Constantin IX Monomaque, in: TM 6 (1976) 223-243. Psellos' Lehrveranstaltungen nach Fuchs, Schulen (s. Anm. 50), 31: „Grammatik, Klassikerlektüre mit Allegorese, Stilkritik und Behandlung der Realien, Rhetorik, Dialektik, Arithmetik, Geometrie, Astronomie, Musik, Physik, Metaphysik und Theologie“. 
juristischen Schriften (die besonders das Eherecht betreffen ${ }^{53}$ ) werden geschätzt; seine Schriften enthielten Kommentare zu Aristoteles. ${ }^{54}$ In seiner Grabrede wird Psellos dem Xiphilinos seine Kenntnisse in Logik, Astronomie, Mathematik, Musik sowie aus dem politischen und religiösen Lebensbereich nachrühmen. ${ }^{55}$

Die Gemeinsamkeiten zwischen Psellos und Xiphilinos waren groß. Beide hatten bedeutenden Einfluss als Berater des Kaisers, diese Stellung wurde aber für beide problematisch, weil der Kaiser immer unberechenbarer wurde: ${ }^{56}$ Seine Günstlinge konnten nicht mehr sicher sein, dass er sie gegen die Angriffe in Schutz nahm, die sich mehrten: Ihr kometenhafter Aufstieg hatte heftigen Protest hervorgerufen; ${ }^{57}$ Psellos verteidigte Xiphilinos: Ophrydas hatte den Vorwurf erhoben, dass Xiphilinos unter Überspringen aller Aufstiegsstufen der Juristenkarriere zum Nomophylax aufgestiegen war. ${ }^{58}$ Psellos wurden z. B. astrologische Praktiken vorgeworfen; zu seiner Umstrittenheit trug seine starke Berücksichtigung Platons und des Neuplatonismus in seiner Lehre bei. Schließlich musste er vor dem Kaiser ein Glaubensbekenntnis ablegen. ${ }^{59}$

Angesichts dieser Schwierigkeiten reifte in beiden der Plan, ins Kloster zu ge-

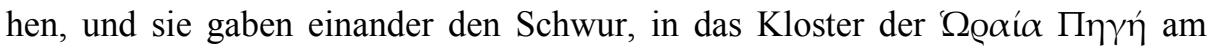
Bithynischen Olymp einzutreten. 1054 macht Xiphilinos den Schwur wahr, ${ }^{60}$ aber Psellos fällt der Schritt schwer, u. a. weil er dem Werben und Drohen des Kaisers ausgesetzt ist, der schon auf seinen Ratgeber Xiphilinos verzichten muss. ${ }^{61}$ Als er

53 Zu Xiphilinos' juristischen Werken s. Wolska-Conus, Les écoles de Psellos (s. Anm. 52), bes. $13-53$.

54 Nicht erhalten sind Traktate über philosophische Probleme im Sinne der Parva naturalia (H.-G. Beck, Kirche und theologische Literatur im byzantinischen Reich [HdAW XII, Byzantinisches Handbuch 2, 1], München ${ }^{2} 1977,756$.)

55 A. Sideras, Die byzantinischen Grabreden. Prosopographie, Datierung, Überlieferung. 142 Epitaphien und Monodien aus dem byzantinischen Jahrtausend (WBS XIX), Wien 1994, 146 Anm. 330.

56 Michele Psello, Imperatori di Bisanzio (Cronografia), Volume I (Libri I-VI 75). Introduzione di D. del Corno, Testo critico a cura de S. Impellizeri, Commento di U. Criscuolo, Traduzione di S. Ronchey, Mailand 1984, VI 191-200 (II 138-150); die Entwicklung wird ausführlich nachgezeichnet von Misch, Autobiographie (s. Anm. 38), III 2,2, 791-795. (Vgl. Weiß, Beamte [s. Anm. 51], 87.)

57 Belege bei P. Joannou, Psellos et le monastère toṽ Náooov, in: BZ 44 (1951) 283-290, hier 284 Anm. 14.

58 G. T. Dennis (Hrsg.), Michaelis Pselli Orationes Panegyricae, Stuttgart/Leipzig 1994, or. 3.

59 Ediert von A. Garzya, On Michael Psellus' Admission of Faith, in: EEBS 35 (1966-67) 41-46, Text 44-46, s. Hunger, Literatur (s. Anm. 37), I 375 mit Anm. 221. Datiert auf 1054: kurz vor dem Klostereintritt (Garzya); F. Tinnefeld, Psellos, Konstantinos, in: TRE 27 [1997] 637-639).

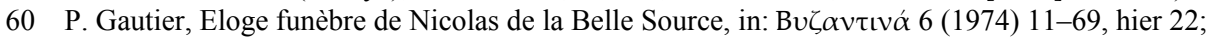

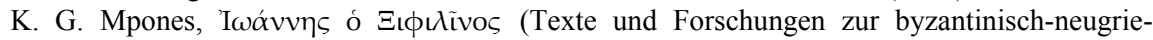
chischen Philologie 24), Athen 1937, 71-76.

61 Michael Psellos' Enkomion auf Xiphilinos in: Sathas MB IV (s. Anm. 38), 438-40. Vgl. Misch, Autobiographie (s. Anm. 38), III 2,2, 795. 
sich schließlich, noch in Konstantinopel, ${ }^{62}$ der Tonsur unterzieht, schickt sich der Kaiser erstaunlich gut in diesen Entschluss; ${ }^{63}$ daraufhin fällt es Psellos noch schwerer, bald auf den Bithynischen Olymp nachzukommen, wie er geschworen hatte. Von Psellos' Zwiespalt legt ein Brief an Xiphilinos Zeugnis ab. ${ }^{64}$

Nach dem Tod des Kaisers am 11. Januar 1055 begibt sich endlich auch Psellos auf den Bithynischen Olymp; ${ }^{65}$ er verlässt freilich das Kloster noch im selben Jahr. ${ }^{66}$ Man begreift, dass er sich in der Umgebung ungebildeter Mönche nicht lange wohl fühlte, man kann sich eine Störung der Harmonie mit Xiphilinos vorstellen; ${ }^{67}$ man hat aber auch den Verdacht, dass Psellos seine Fühler ausgestreckt hatte und bereits die Zeit für gekommen hielt, am Kaiserhof, d. h. bei der nun allein regierenden Theodora, die nächste Vertrauensposition anzustreben. ${ }^{68}$ Auch Xiphilinos wird nicht für sein ganzes Leben in Bithynien bleiben; man wird ihn im Jahre 1064 auf den Patriarchenstuhl von Konstantinopel holen, und zwar auf Betreiben des Psellos; so ist die Auffassung des Psellos von Freundschaft. ${ }^{69}$ Psellos wird sich als wendiger Hofmann bis zum Beginn der Herrschaft Kaiser Michaels VII. am Kaiserhof halten. Vielleicht gab es die ironische Pointe, dass ihn sein Weg gegen seinen Willen noch einmal eine Zeitlang ins Kloster führte, ${ }^{70}$ aber es sind noch nicht alle Zweifel an diesen Thesen beseitigt. ${ }^{71}$

62 Man rechnet damit, dass er erst nach dem Tod des Kaisers auf den Bithynischen Olymp geht (so R. Volk, Der medizinische Inhalt der Schriften des Michael Psellos [MBM 32], München 1990, 438, Weiß, Beamte [s. Anm. 51], 88).

63 Michael Psellos Chronographia VI 199 (II 148) (s. Anm. 56).

64 E. V. Maltese, Epistole inedite di Michele Psello I-II, in: Studi italiani di filologia classica, 3. ser. 5 (1987) 82-98, 214-223, bes. 96-98 (Michael Psellos ep. 5), vgl. G. Weiß, Forschungen zu

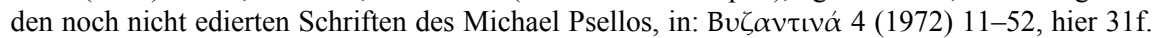
Die Situation wird ähnlich geschildert in Michael Psellos' Enkomion auf Xiphilinos in: Sathas MB IV (s. Anm. 38), 438.

65 Wahrscheinlich zweite Hälfte Januar 1055: Gautier, Eloge (s. Anm. 60), 22, ebenso Hunger, Literatur (s. Anm. 37), I 375.

66 Agapitos, Teachers (s. Anm. 33), 181 nimmt eine Rückkehr nach Konstantinopel bereits im Frühjahr 1055 an, geht aber von einem Wechsel auf den Bithynischen Olymp bereits vor dem Tod des Konstantinos Monomachos aus.

67 Hunger, Literatur (s. Anm. 37), I 375.

$68 \mathrm{Zu}$ Theodora s. K.-P. Todt, Die Frau als Selbstherrscher: Kaiserin Theodora, die letzte Angehörige der makedonischen Dynastie, in: JÖB 50 (2000) 139-171, 163: Psellos war schon zu Lebzeiten des Monomachos Theodoras Geheimsekretär gewesen. Nach Volk, Inhalt (s. Anm. 62), 31 ruft ihn Theodora noch 1055 zurück; s. a. Misch, Autobiographie (s. Anm. 38), III 2,2, 796.

69 F. Tinnefeld, „Freundschaft“ in den Briefen des Michael Psellos. Theorie und Wirklichkeit, in: JÖB 22 (1973) 151-168. Ein Beispiel für die Wandelbarkeit von Psellos' Freundschaft zeichnet E. de Vries-van der Velden, Les amitiés dangereuses: Psellos et Léon Paraspondylos, in: BS1 60 (1999) 315-350.

70 Vertreten wird einerseits die These, dass ihm das Kloster Toũ Nógoov zum Gefängnis wurde Joannou, Psellos (s. Anm. 57), passim, bes. 286-289 -, andererseits, dass er zurück in das Klos-

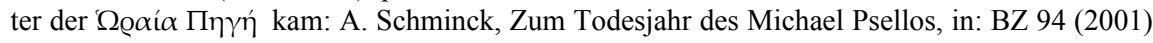


Der „Humanistenbrief“ des Psellos an Xiphilinos wird jetzt in die Zeit datiert, kurz nachdem Psellos das Kloster wieder verlassen hat, also 1056 oder noch 1055; Xiphilinos ist im Kloster auf dem Olymp geblieben (auf das Patriarchenamt, das Xiphilinos von 1065 an innehatte, wird nirgends angespielt). Auf diese Situation zielt es, wenn Psellos die Frage verhandelt, ob der Aufenthalt auf dem Berg, dem mönchischen Ort schlechthin, ${ }^{72}$ eine hinreichende oder auch nur notwendige Bedingung moralischer Unanfechtbarkeit ist. Psellos verficht die Ansicht, dass der Aufenthaltsort, Berg oder Stadt, keinen Wert und keine Garantie darstellt; der moralische Wert eines Menschen hängt von der Entscheidung seines freien Willens ab. So argumentiert nicht der, der als Mönch auf dem Berg lebt, sondern der, der ein Leben in der Stadt rechtfertigen will. Wirklich bezeichnet

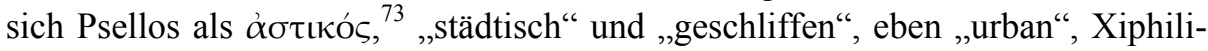
nos ist es offenbar, der den Vorrang des Berglebens per se vertritt.

Psellos nimmt aus Xiphilinos' Brief die Formulierung auf, die ihm so sehr nach Drohung mit der „Inquisition“ klang: „Wenn Du mir nun gehorchen willst damit ich dich bis hierher ${ }^{74}$ nach deiner eigenen Manier anspreche -, so bilde dir nichts ein auf den Rückzug auf den Berg, und schwelge nicht in einem Leben, das nicht in Kunst und Wissenschaft schwelgt; sondern wenn es irgendwo eine Ebene, ein ganz breites Tal oder eine zerklüftete Schlucht oder ein unsagbares und untersagtes Erdloch gibt, dann begib dich dort hinab und verkrieche dich, um dich über alle Bücher zu beugen, die unsrigen wie die heidnischen. ${ }^{\text {" }} 75$

Für sich hingegen entwirft Psellos das Leben, das er von nun an führen will: ${ }^{76}$ Er will bald die Überlieferung der großen Eremiten studieren, bald seinen ge-

190-196. (Ioannou gibt 1063 als Enddatum an, legt jedoch kein genaues Anfangsdatum fest, nur, dass es nach dem Aufenthalt auf dem Bithynischen Olymp war).

71 Gegen einen Aufenthalt im Kloster Toũ Nó@oov Weiß, Forschungen (s. Anm. 64), 100 und Anm. 323. Direkt gegen Schminck, Todesjahr (s. Anm. 70) A. Karpozilos, When did Michael Psellus die?, in: BZ 96 (2003) 671-677, hier 676 mit Anm. 26: $\mu \alpha \kappa \alpha \varrho \iota \omega \tau \alpha \tau o \varsigma$ impliziere nicht, dass Psellos ,jüngst verstorben“ sei.

72 Zum Bergmotiv im Mönchtum s. K. Metzler, Eustathios von Thessalonike und das Mönchtum. Untersuchungen und Kommentar zur Schrift „De emendanda vita monachica“ (Supplementa Byzantina 9), Berlin/New York 2006, 248f. (Register mönchischer Kernbegriffe s. v. őoo und andere Bergesmetaphorik).

73 Michael Psellos ep. ad Xiph. 123 Criscuolo (s. Anm. 38).

74 D. h. ohne die implizite Drohung von Xiphilinos' ursprünglicher Formulierung.

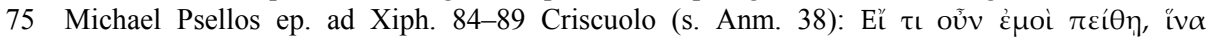

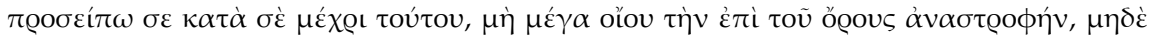

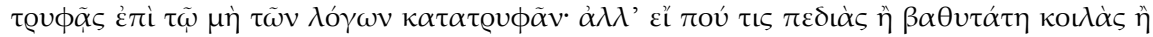

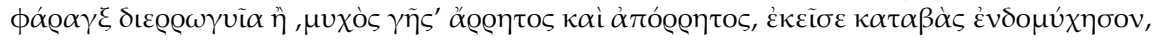

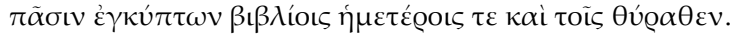

76 Michael Psellos ep. ad Xiph. 205-17 Criscuolo (s. Anm. 38). Der Brief kann also nicht geschrieben sein, bevor Psellos auf den „Berg“ kam; denn er schwankt nicht mehr, wie das für die Zeit vor seinem Verlassen Konstantinopels belegt ist (gegen Weiß, Forschungen [s. Anm. 64], 31f.). 
wohnten Studien nachgehen: Logik, Naturphilosophie, Geschichtsschreibung, Metaphysik. ${ }^{77}$ Für dieses Programm ist er freilich in der Hauptstadt mit ihren Bibliotheken besser gerüstet als in einem entlegenen Kloster. Dass dieses Leben nicht sehr von dem abweicht, das er früher geführt hat, hält ihn nicht davon ab, sich weiterhin als Mönch zu fühlen (was andere anders beurteilen).

Aus der Situation, dass Psellos das Kloster bereits wieder verlassen hat, wird schlagartig der Streitpunkt der beiden Kontrahenten im Hintergrund klar: Xiphilinos hat Psellos vorgehalten, dass er das Kloster verlassen hat und zu seiner paganen Bildung in die Kaiserstadt zurückgekehrt ist - ein Vorwurf, der Psellos auch von anderer Seite gemacht wurde $;^{78}$ Xiphilinos wollte geltend machen, dass das gewählte Klosterleben moralisch höher stehe und dass die Beschäftigung mit paganer Philosophie dem christlichen Denken nur Abbruch tun könne; dabei scheint ihm die Formulierung „dein Platon“ unterlaufen zu sein. Geschickterweise reagiert Psellos zuerst und zuletzt auf den damit erhobenen Platonismus-Vorwurf, der sich verschieden wenden lässt, während er sein persönliches Verhalten, dass er das Kloster vor Jahresfrist wieder verlassen hat, lieber nicht zu detailliert zur Sprache bringt. Wenn aber das so ist, gewinnt die Diskussion über Platons Stellenwert etwas den Charakter eines Scheingefechts, in dem es nicht darum ging, wie gefährlich die Beschäftigung mit Platon in Byzanz werden konnte. Aber ungefährlich war sie auch nicht. ${ }^{79}$

In eine ganz andere Welt sieht man sich versetzt, wenn man sich dem Staatsmann und Gelehrten Theodoros Metochites zuwendet, wie er im 14. Jahrhundert darüber nachdenkt, welchen Ort zu seiner Zeit Bildung hat - eine Bildung, die nicht anders gedacht werden kann als unter Einschluss des paganen Erbes. Man kennt den Lebensweg des Theodoros Metochites: den glanzvollen Aufstieg vom Sohn eines Verfemten ${ }^{80}$ zum höchsten Minister, sein vielfältiges Wirken als Staatsmann, Gelehrter, Literat und Stifter, den tragödienreifen Sturz vom Gipfel der Macht; es sei hier auch nicht das ganze Personal der Auseinandersetzungen einzeln vorgestellt, die Konstellation von Theodoros Metochites und Nikephoros Chumnos ist der von Psellos und Xiphilinos zu ähnlich; festgehalten sei nur, dass sich auch und gerade bei Theodoros der Dialog-Charakter der Thesen belegen

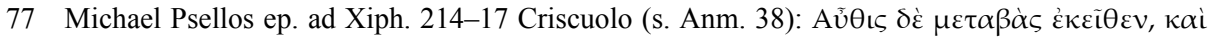

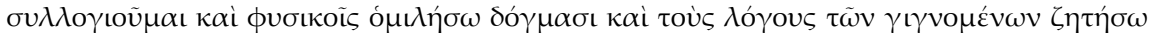

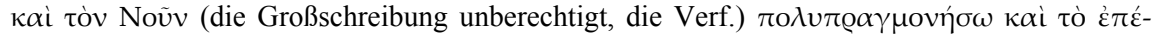

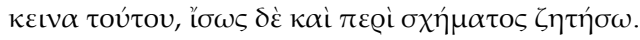

78 Joannou, Psellos (s. Anm. 57), 285 Anm. 16 führt an: den Mönch Pherebios (Sathas MB V 424 428), den Mönch Iakobos, den er dann als Trunkenbold verspottete, einen Mönch des SabasKlosters, Ioannes Patrikios, den Abt des Olymps. Vgl. Joannou, Psellos, 286 Anm. 9. S. a. Misch, Autobiographie (s. Anm. 38), III 2,2, 796.

79 S. S. 295, Anm. 49.

80 Zur Vita des Vaters Georgios Metochites s. Beyer, Studien (s. Anm. 37), 19-21. 
ließe. Herausgegriffen seien aus verschiedenen Schriften Beispiele dafür, wie Theodoros mit dem antiken Erbe umgeht. Es ist kein Zufall, dass das Bild impressionistisch wird: Anders als mit gegensätzlichen Bruchstücken kann man kein Bild von Metochites gewinnen, für den ein Nebeneinander von traditionellen Topoi und neuen Perspektiven in originellen Fragestellungen charakteristisch ist.

Mit größter Selbstverständlichkeit werden die aktuellen Streitfragen im Material der Antike verhandelt: Wenn Metochites sich z. B. gegen den Vorwurf wehrt, sein Stil weise keine Klarheit auf, so beruft er sich auf den anerkannten Rang des harschen Demosthenes. ${ }^{81}$ Der Gegner (besagter Nikephoros Chumnos), der Metochites treffen will, wirft ihm in seinem eigenen Spezialgebiet, der Astronomie, eine verkehrte Platon-Auslegung vor; Metochites wehrt sich mit dem Vorwurf, Chumnos verstehe nichts von Platon. (Freilich ist Theodoros selbst bei seinen Platon-Zitaten von Iamblich abhängig. ${ }^{82}$ ) Die aus der heidnischen Antike ererbte Literatur ist die Welt, in der Metochites lebt - seine hohe Stellung in der Politik ist mit ihr nicht nur verträglich, sondern von ihr geradezu abhängig; ${ }^{83}$ dennoch, so verwoben er darein ist, gewinnt er an manchen Stellen eine erstaunliche Distanz zu ihr.

Das zeigt sich, wenn Theodoros zugleich Bildung, wie es Konvention ist, als hohes Gut preist und ihr (im Gegensatz zum platonisierenden Joseph Rhakendytes) doch abspricht, das höchste Gut zu sein. Eine frühe ${ }^{84}$ Schrift, der Ethikos, ist zunächst einmal ein Protreptikos, die Werbung bei einem ungenannten jungen Mann für größere Sorgfalt bei den Bildungsbemühungen. Metochites preist die Bildung - nicht entscheidend anders als Basileios - für ihre ethische Lebenshilfe: $\pi \alpha \iota \delta \varepsilon i ́ \alpha$ hilft, Schicksalsschläge zu überwinden. ${ }^{85}$ Doch wenn Metochites von Bildung spricht, beschränkt sich das nicht auf die Kunst des Wortes; der große Staatsmann hält auch politische Fähigkeiten für nötig, die er auch Privatleuten zuspricht. Das ist eine gehörige Relativierung der Bildung im traditionellen Verständnis und widerspricht der Abwertung des Leiblich-Praktischen, wie sie von stärker platonisierenden Zeitgenossen vertreten wird. ${ }^{86}$

81 H. Hunger, Theodoros Metochites als Vorläufer des Humanismus in Byzanz, in: BZ 45 (1952) 4-19, hier 17.

82 Metochites' Platon-Zitate zur Stützung seiner Astronomie stammten größtenteils von Iamblichos, eine Epinomis-Stelle sei gefälscht, so referiert bei Beyer, Studien (s. Anm. 37), 10.

83 M. Hinterberger, Studien zu Theodoros Metochites. Gedicht I - Des Meeres und der Liebe Wellen - Die Angst vor dem Neid - Die autobiographischen Texte - Sprache, in: JÖB 51 (2001) 285-319, hier 287f.

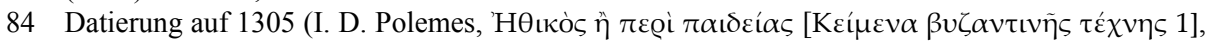
Athen 1995, 9).

85 Hunger, Metochites (s. Anm. 81), 14.

86 Die monastische Position wird hier (wahrscheinlich) vertreten von Thomas Magistros und Ioannes Zacharias, Polemes in Polemes, 'HӨıkò einandersetzung mit Nikephoros Chumnos um Platon: s. Hunger, Literatur (s. Anm. 37), I 22f. 
Hier setzt Theodoros Metochites neue Akzente im Streit um das Verhältnis

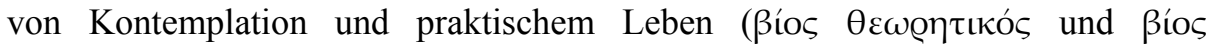

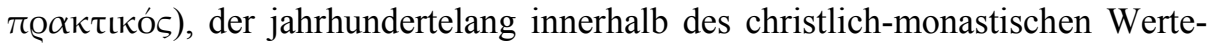
systems geführt worden ist, indem er an das klassisch-antike Modell anknüpft. Metochites ist immer wieder für Überraschungen gut, wenn es um originelle Akzentsetzungen bei alten Problemen geht. Wenn er von Platon und Aristoteles spricht, kommt kein Zweifel daran auf, wie sehr er ihre Größe anerkennt; mit ihrer Eitelkeit und ihren Eifersüchteleien geht er dennoch ausgesprochen kühl ins Gericht.$^{87}$ Er beschränkt nicht, wie es sein Bildungsgang nahelegen würde, seinen Gesichtskreis auf die klassische griechische Geschichte; er sieht keinen Grund, andere Kulturen für weniger wichtig zu halten - zur Verewigung der griechischen fanden sich lediglich die besten Schriftsteller. Er ist ganz und gar nicht wehleidig darin, seinen eigenen Standpunkt dem Zweifel auszusetzen, dafür noch ein Beispiel: Das (späte) Werk der $\Sigma \eta \mu \varepsilon เ \omega ́ \sigma \varepsilon เ \varsigma \gamma \nu \omega \mu \iota \kappa \alpha i^{88}{ }^{88}$ geläufig unter dem lateinischen Titel „Miscellanea“, setzt mit einer merkwürdigen These ein: ${ }^{89}$ „dass es unmöglich ist, jetzt etwas zu sagen“, d. h.: in heutiger Zeit etwas Neues, Gültiges zu sagen. Ist nicht alles schon gesagt, und zwar auf die gültigste Art gesagt? Ausdrücklich ist das ein Zeitproblem, die Ungnade der späten Geburt: Gleich mit dem zweiten Satz wird als Beispiel Cato angeführt, der trotz höchster Fähigkeiten und Leistungen das Konsulat verfehlte, weil er nicht in der richtigen Zeit (sondern gegen Ende der Republik) lebte $;^{90}$ Theodoros ist überzeugt, ein Spätgeborener zu sein: ${ }^{91}$ „Aber für uns, die wir im Leben die Erfahrung machen, dass wir spät in der Geschichte leben, ist es nicht möglich, auf irgendeine Art die Rede zu gebrauchen, wenn es sich überhaupt trifft, dass einer das Zeug dazu hat.“

Doch müsste man nicht verstummen, wenn man diese Frage stellt? Für Metochites ist sie aber die Einleitung in ein langes Werk; wenn das Proömium zu seinem Ende kommt, ist es schon das erste Kapitel einer langen Reihe von Essays

87 Aristoteles' Eitelkeit: K. Hult, Theodore Metochites on Ancient Authors and Philosophy. Semeioseis gnomikai 1-26\&71. A Critical Edition with Introduction, Translation, Notes, and Indexes (Studia Graeca et Latina Gothoburgensia 65), Göteborg 2002, Misc. 5; Platon und Aristoteles gingen mit ihren Vorgängern respektlos um: Misc. 10.

88 Zur Rekonstruktion des Titels s. Hult, Theodore Metochites (s. Anm. 87), XIVf.

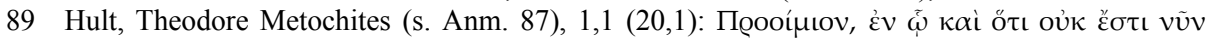
$\lambda \varepsilon ́ \gamma \varepsilon เ v: A^{\prime}$.

90 Verständnis nach Hult, Theodore Metochites (s. Anm. 87), 21 Anm. 2.

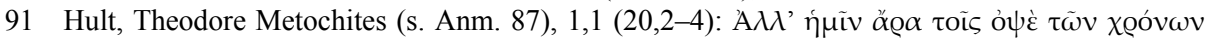

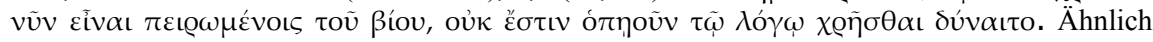

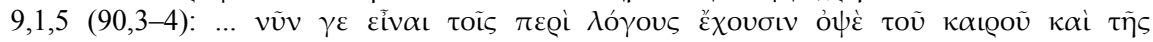

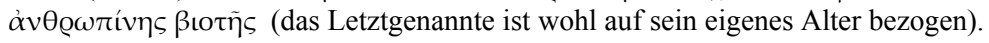


und Betrachtungen gewesen. ${ }^{92}$ Der Leser mag sein Urteil fällen, woraus Metochites die Sicherheit bezog, dennoch Neues zu sagen. ${ }^{93}$

Haben wir an den drei Autoren eine Konstante oder gar eine Entwicklungslinie $^{94}$ für die byzantinische Kultur erkannt? Man kann den Einwand dagegen erheben: Die Texte antworten nicht aufeinander und nicht auf dieselbe Fragestellung. Aber es zeigt sich doch eine Konstante: dass sich zu allen Zeiten der byzantinischen Geschichte Probleme der Adaption der paganen Bildung in einer christlichen Kultur stellten, und zwar im Dialog mit zeitgenössischen Gegnern, in Antwort auf die aktuellen Fragen. Diese stellten sich zu verschiedenen Zeiten verschieden: in der Zeit der Konsolidierung des Christentums in der Frage, ob und wenn ja, wie - man den traditionellen Bildungsweg, der auch ein Karriereweg für die gesellschaftliche Elite war, in den neuen Glauben einbeziehen sollte; in mittelbyzantinischer Zeit hat Michael Psellos Anlass, seine Beschäftigung mit dem paganen Erbe zu rechtfertigen; in spätbyzantinischer Zeit ist für Theodoros Metochites die Beschäftigung ohne jede Anfechtung außer den geistigen Hindernissen, die ihr selbst entspringen, doch er muss sein Selbstbewusstsein gegen das bedrückend autarke antike Erbe behaupten.

Gerade in der Verschiedenheit der Situation belegen alle drei Autoren die Konstante, dass in ganz verschiedenen Situationen immer wieder Fragen gehört wurden, auf die sich mit Hilfe des klassischen Erbes neue Antworten geben lie-

92 Metochites' Lösung der Aporie ist, dass er Gedanken zu Fragen veröffentlicht, von denen er annimmt, sie müßten auch anderen missfallen haben, Hult, Theodore Metochites (s. Anm. 87), $1,3.5-7(24,23-26,12)$.

93 Auf der einen Seite steht Hans-Georg Becks Auffassung (H.-G. Beck, Theodoros Metochites. Die Krise des byzantinischen Weltbildes im 14. Jahrhundert, München 1952, 24), Metochites vertrete, trotz der Aporie, den Anspruch, auch jetzt noch etwas zu sagen zu haben, nämlich durch die Veröffentlichung persönlicher, unrhetorischer, bekenntnishafter Aufsätze. Auf der anderen Seite steht seit kurzem Bydéns Analyse (B. Bydén, The Nature and Purpose of the Semeioseis gnomikai: The Antithesis of Philosophy and Rhetoric, in: Hult, Theodore Metochites [s. Anm. 87], 245-288 passim, 251-258: zum Titel; 263-269: zu Gedicht 12; 269-273: zu dem Brief von Nikephoros Gregoras (Nicephori Gregorae epistulae ed. P. A. M. Leone, Matino 1982, II epist. 23), nach der die $\Sigma \eta \mu \varepsilon t \omega ́ \sigma \varepsilon เ \varsigma$ keinesfalls eine zweckfreie Zusammenstellung privater Notizen seien; ihre Neuheit und damit Metochites' Lösung der Aporie bestehe hingegen im Formalen: im auf Privatheit und Aufrichtigkeit hin stilisierten Ausbreiten assoziativ verknüpfter, für das Werk konzipierter Essays (Bydén hat die Semantik der für diese Frage heranzuziehenden Zeugnisse genau untersucht und die Alternative zugespitzt).

Demgegenüber wird sich die Fülle der Beobachtungen Becks trotz seiner zeitgebundenen existentialistischen (dazu Beyer, Studien [s. Anm. 37], 9 gegen E. de Vries-van der Velden, Théodore Métochite: Une réévaluation, Amsterdam 1987) Fragestellung und Terminologie sinnvoll erweisen; damit wird das besondere Profil der Ansichten des Metochites auch wieder zur Geltung kommen. Bydén, Nature, 251 sieht selbst Raum für die Anerkennung von Becks Sicht.

94 Von Entwicklung wird man für Byzanz nur mit äußerster Vorsicht sprechen. Und gewiss handelt es sich nicht um eine Entwicklung, die dazu geführt hätte, dass es keine Frage mehr war, dass ein Christ sich mit Platon beschäftigen dürfe - man denke etwa an die Gegner Plethons. 
ßen; dass sich immer aufs Neue unvergleichliche Persönlichkeiten auf ganz individuelle Art mit dem paganen Erbe auseinandersetzen und immer wieder eigene Akzente setzen. So ist es keine unendliche Geschichte, aber eine lange und fruchtbare; nicht 1100 Jahre Niedergang, sondern eine Folge von immer neuen Renaissancen. 
